\title{
Dynamic Power Control and Optimization Scheme for QoS-Constrained Cooperative Wireless Sensor Networks
}

\author{
Ziqiang Feng, Ian Wassell \\ Computer Laboratory \\ University of Cambridge, UK \\ Email: \{zf232, ijw24\}@cam.ac.uk
}

\begin{abstract}
Cooperative transmission can significantly reduce the power consumption associated with long distance transmission in wireless sensor networks (WSNs). In this paper, we analyze the optimal power consumption of cluster-based multi-hop transmission for a cooperative WSN. With specific Quality of Service (QoS) constraints on delay and channel capacity, we show that the power optimization problem of the whole network has no closed-form solution in a slow flat Rayleigh fading environment. Thus we propose a dynamic power control and optimization (DPCO) scheme that can jointly determine the optimal number of cooperative sensors and their transmission power. We further propose a channel approximation algorithm that can significantly reduce the computational complexity of the DPCO scheme.
\end{abstract}

\section{INTRODUCTION}

Energy optimization is an important issue in wireless sensor networks (WSNs) where the low-power sensors are expected to operate for many years without battery replacement. Furthermore, certain applications may have Quality of Service $(\mathrm{QoS})$ requirements on delay and channel capacity. For instance, it is important for the sink node to receive data in a timely manner in an industrial control system [1]. Traditional energy optimization techniques may not always guarantee the QoS requirements in WSNs, especially in fading environments.

It has been proved that multi-antenna system use less energy for data transmission in fading channels compared to singleantenna systems [2]. However, a multi-antenna system requires complex transceiver circuits that are not practical for low-cost wireless sensors. Cooperative multiple-input-multiple-output (MIMO) [3] and virtual MIMO techniques [4] are proposed to enable MIMO techniques to be utilized in WSNs. For the single-hop cooperative MIMO systems analyzed in [3], it is shown that for long-distance transmission, they are more energy efficient compared to a single-antenna system. In [5], multi-hop cooperative MIMO channels are analyzed, where the design target is to minimize the outage probability given an energy constraint and target outage channel capacity. However, the minimum outage probability may not give the maximum average outage channel capacity [6]. Minimizing the outage probability may also lead to full-power operation which significantly decreases the lifetime of the WSN.

In this paper, we investigate the energy consumption in a cluster-based multi-hop cooperative WSN with QoS requirements on delay and channel capacity. We mainly focus on the physical layer optimization and we assume that the clustering and routing problems are handled by upper layers. The multi-hop transmission consists of several single-hop cluster-to-cluster transmissions. Each single-hop transmission consists of three phases, namely the Preparation Phase (PP), the Broadcast Phase (BP) and the Cooperation Phase (CP). In the PP, the cluster head of the transmitter cluster calculates the transmission power to be used in the BP and CP. In the BP, the cluster head broadcasts the data along with the power control message in the transmitter cluster. In the $\mathrm{CP}$, all nodes of the transmitter cluster that received the data successfully in the BP cooperatively transmit the data to the cluster head of the receiver cluster through a multiple-input-single-output (MISO) channel. For intra-cluster transmission in the BP, we assume that all nodes in the cluster have perfect transmit and receive channel state information (CSI). For inter-cluster transmission in the $\mathrm{CP}$, we assume that nodes in the receiver cluster know the CSI and that nodes in both clusters know the distribution of the CSI. Note that our MISO approach can be extended to a MIMO approach by utilizing selection diversity in the receiver cluster [5].

To minimize the total energy consumption, we propose a dynamic power control and optimization (DPCO) scheme that can optimize the total energy consumption without violating the QoS requirements. Given the number of cooperative nodes as an input condition, the scheme first calculates the conditional optimal power consumption using the dynamic power control (DPC) algorithm. Then it determines the optimal number of cooperative sensors using the results of conditional optimal power consumption. We show that the DPC algorithm converges to the optimum in $O(1)$ iterations. We further propose a channel approximation algorithm that can significantly reduce the computational complexity of the DPCO scheme.

The rest of the paper is organized as follows: Section II describes the system model of the multi-hop cluster-based cooperative WSN and expresses the QoS requirements. The DPCO scheme is proposed in Section III. We further propose an approximation algorithm to reduce the computational complexity of the DPCO scheme. Section IV shows the simulation results of the proposed scheme. Finally, we conclude the paper in Section V.

\section{System Model}

We consider a multi-hop cluster-based WSN. The transmission between two adjacent clusters is defined as a single- 


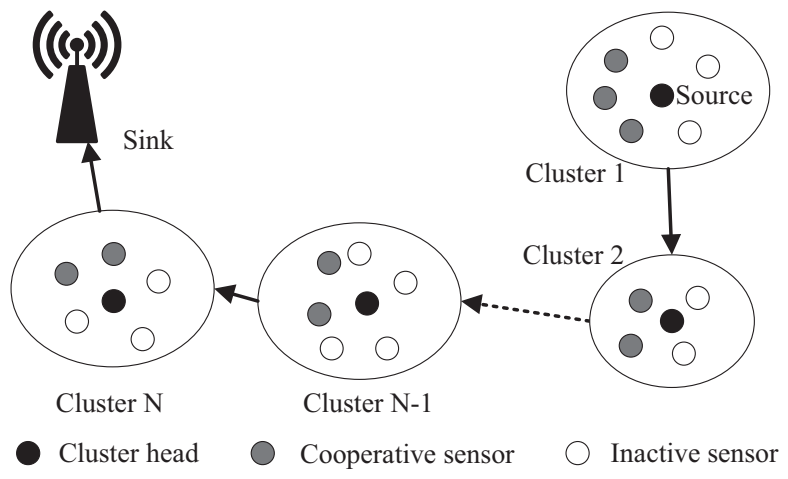

Fig. 1. Multi-hop cluster-based cooperative wireless sensor network

hop transmission. In our model, we assume that the nodes are grouped into $N$ clusters and we have $N-1$ hops during the transmission. The routing path is predefined. Before the transmission, each cluster selects a node as its cluster head. Efficient clustering and routing algorithms such as those in [7], [8] can be used. The cluster head of cluster 1 is assumed to be the source node. The system model of the cooperative WSN is shown in Fig. 1.

\section{A. Transmission Scheme}

The transmission between adjacent clusters has three phases, namely the preparation phase (PP), the broadcast phase (BP) and the cooperation phase (CP). The time duration of the three phases are $\lambda_{1} T, \lambda_{2} T$ and $\lambda_{3} T$ respectively where $\lambda_{1}+\lambda_{2}+\lambda_{3}=1$ and $T$ is the time duration of each single-hop transmission. We assume that each cluster contains $n$ sensors that are uniformly deployed in the cluster and the cluster head is located near the center of the cluster.

1) Preparation Phase: In the PP, the cluster head in the transmitter cluster calculates the optimal number of cooperative nodes $\left(n_{t r}\right)$, the transmission power in the BP $\left(P_{b t}\right)$ and $\mathrm{CP}\left(P_{c t}\right)$ using the DPCO scheme. The value of $n_{t r}, P_{b t}$ and $P_{c t}$ are carried in the power control message and sent to other cooperative nodes along with the data in BP.

2) Broadcast Phase: In the BP, the cluster head broadcasts the data along with the power control message to the cooperative sensors within its cluster with power $P_{b t}$. The sensors that received the data successfully are selected to transmit with the cluster head in the CP. Since we assume that the sensors in the cluster are uniformly deployed and the cluster head is located in the center of the cluster, the number of the active sensors is proportional to $P_{b t}$ and the total number of sensors $n$ in each cluster. By selecting an appropriate level of $P_{b t}$ to broadcast the data, we can have $n_{t r}$ nodes selected as the cooperative nodes (including the cluster head itself) in the CP.

3) Cooperation Phase: In the $\mathrm{CP}$, the cooperative nodes jointly transmit the data to the cluster head in the receiver cluster using an orthogonal Space-Time Block Code (STBC). We assume that the number of cooperative nodes is $n_{t r}$, including the cluster head itself. The total transmission power $P_{c t}$ is equally divided among the cooperative nodes. The transmission power used by each sensor is consequently $\frac{P_{c t}}{n_{t r}}$.

\section{B. QoS Requirements}

We consider a multi-hop cluster-to-cluster communication scheme containing $N$ clusters (see Fig. 1). We assume the cluster head is located in the center of each cluster. The distance between the centers of two adjacent clusters is denoted by $d$. We also assume long-range transmission between adjacent clusters since cooperative transmission is more energy efficient in that case [3]. Therefore the distances between the sensors in the adjacent clusters are approximately equal. We modeled the channel in the BP and CP using the additive white Gaussian noise (AWGN) channel and slow flat Rayleigh fading channel with AWGN respectively, since the inter-cluster distance is much larger than the intra-cluster distance [3].

Without loss of generality, we consider the transmission between cluster $m$ and cluster $m+1$. In the $\mathrm{CP}$, the cooperative sensors jointly transmit the data to the next cluster head using STBC through a MISO channel. The received signal at the cluster head of cluster $m+1$ is given by:

$$
Y_{m+1}=\sqrt{\Lambda} H X_{m}+N_{m+1}
$$

where $\Lambda=\beta d^{-\alpha}$ is the path loss, $\beta$ is the path loss constant related to the channel and antennas (i.e., the wavelength of the signal and the antenna gain) and $\alpha$ is the path loss exponent. The elements of the $1 \times n_{t r}$ channel vector $H$ are independent identical distribution complex Gaussian random variables with zero mean and unit variance. $X_{m}$ is the $n_{t r} \times l$ transmitted signal with transmission power $\frac{P_{c t}}{n_{t r}}$, where $l$ is the length of the STBC. $N_{m+1} \sim N\left(0, \sigma^{2}\right)$ is the $l \times 1$ AWGN at the receiver. The transmission power of the transmitted space-time codeword is $\frac{P_{c t}}{n_{t r}}$ per node.

Considering the nodes in the transmitter cluster only know the distribution of the CSI, the outage capacity $C_{\text {out }}[\mathrm{bps} / \mathrm{Hz}]$ of the $\mathrm{CP}$ is defined as [6]:

$$
C_{\text {out }}=\lambda_{3} \log _{2}\left(1+\gamma_{\text {out }}\right),
$$

where $\gamma_{\text {out }}$ is the minimum signal-to-noise ratio (SNR) for successful decoding at the receiver side. The outage probability $p_{\text {out }}$ is defined as:

$$
p_{\text {out }}=\operatorname{Pr}\left(\gamma<\gamma_{\text {out }}\right),
$$

where $\gamma$ is the actual SNR at the receiver side. According to our channel model in (1), $\gamma$ is given by:

$$
\gamma=\frac{\Lambda P_{c t}\|H\|_{F}^{2}}{n_{t r} \sigma^{2}},
$$

where $\sigma^{2}$ is the noise power and $\|H\|_{F}^{2}$ is the squared Frobenius norm of the $1 \times n_{t r}$ channel vector $H$. According to the definition of $H,\|H\|_{F}^{2} \sim \chi^{2}\left(2 n_{t r}\right)$ is a chi-square variable with $2 n_{t r}$ degrees of freedom.

The average outage capacity is given by:

$$
\bar{C}_{\text {out }}=\left(1-p_{\text {out }}\right) C_{\text {out }}=\lambda_{3}\left(1-p_{\text {out }}\right) \log _{2}\left(1+\gamma_{\text {out }}\right) \text {. }
$$

1) Delay Requirement: We consider the delay of the multihop cluster-to-cluster communication using an automatic repeat request (ARQ) protocol. We assume that the outage probabilities are equal in each of the $N-1$ hops. Therefore, 
for transmission through $N-1$ hops, the number of failures $k$ follows the negative binomial distribution:

$$
k \sim N B\left(N-1 ; p_{\text {out }}\right) .
$$

The probability mass function of the negative binomial distribution is

$$
f\left(k ; N-1, p_{\text {out }}\right)=\left(\begin{array}{c}
k+N-2 \\
k
\end{array}\right) p_{\text {out }}^{k}\left(1-p_{\text {out }}\right)^{N-1} .
$$

Proposition 1: Given the delay requirement $D$, the average delay of the transmission through $N-1$ hops is given by:

$$
\bar{T}=E[(k+N-1) T]=\frac{(N-1) T}{1-p_{\text {out }}} .
$$

Thus we can have reliable transmission if $p_{\text {out }} \leq 1-\frac{(N-1) T}{D}$ for any delay requirement $D>(N-1) T$. If $D \leq(N-1) T$, it is impossible to have reliable transmission under the delay requirement.

Proof: We first prove that $E[k]=\frac{(N-1) p_{\text {out }}}{1-p_{\text {out }}}$. Since the number of failures $k$ follows the negative binomial distribution, the mean value of $k$ can be calculated as:

$$
\begin{aligned}
E[k] & =\sum_{k=0}^{\infty} k f\left(k ; N-1, p_{\text {out }}\right) \\
& =\sum_{k=0}^{\infty} \frac{k(k+N-2) !}{k !(N-2) !} p_{\text {out }}^{k}\left(1-p_{\text {out }}\right)^{N-1} \\
& =\frac{(N-1) p_{\text {out }}}{1-p_{\text {out }}} \sum_{k=1}^{\infty} \frac{(k+N-2) !}{(k-1) !(N-1) !} p_{\text {out }}^{k-1}\left(1-p_{\text {out }}\right)^{N} \\
& =\frac{(N-1) p_{\text {out }}}{1-p_{\text {out }}} \sum_{k=0}^{\infty} f\left(k ; N, p_{\text {out }}\right) \\
& =\frac{(N-1) p_{\text {out }}}{1-p_{\text {out }}} .
\end{aligned}
$$

Note that in (8) $N$ and $T$ are constants. Thus the average delay is given by:

$$
\bar{T}=E[(k+N-1) T]=\frac{(N-1) T}{1-p_{\text {out }}} .
$$

For any delay requirement $D>(N-1) T$, we must have $\bar{T}=\frac{(N-1) T}{1-p_{\text {out }}} \leq D$ and thus $p_{\text {out }} \leq 1-\frac{(N-1) T}{D}$ to satisfy the reliable transmission requirement. Note that $0<p_{\text {out }}<1$. If $D \leq(N-1) T$, we should have $p_{\text {out }} \leq 1-\frac{(N-1) T}{D} \leq 0$ for reliable transmission, which is impossible to satisfy.

2) Capacity Requirement: We assume that there are a total of $L$ bits for transmission. In order to have reliable transmission in the $\mathrm{CP}$, we have

$$
\frac{L}{T} \leq R_{c t} \leq \bar{C}_{\text {out }}
$$

where $R_{c t}[\mathrm{bps} / \mathrm{Hz}]$ is the transmission data rate in the $\mathrm{CP}$.

In the $\mathrm{BP}$, the transmission data rate $R_{b t}[\mathrm{bps} / \mathrm{Hz}]$ should be no less than the transmission data rate in the $\mathrm{CP}$. Thus we have

$$
R_{b t} \geq R_{c t} \geq \frac{L}{T}
$$

Proposition 2: For any transmission under the QoS requirement and $D>(N-1) T$, there exists $p_{\text {out }}^{*}$ that can maximize $\bar{C}_{\text {out }}$, which can be denoted as follows

$$
\begin{aligned}
& \bar{C}_{\text {out }}^{*}=\max _{p_{\text {out }}}\left\{\lambda_{3}\left(1-p_{\text {out }}\right) \log _{2}\left(1+\gamma_{\text {out }}\right)\right\} \\
& \text { s.t. } \quad \bar{C}_{\text {out }} \geq \frac{L}{T}, 0<p_{\text {out }} \leq 1-\frac{(N-1) T}{D}
\end{aligned}
$$

where $\gamma_{\text {out }}=\frac{\Lambda P_{c t} F^{-1}\left(p_{\text {out }} \mid 2 n_{t r}\right)}{n_{t r} \sigma^{2}}$ and $F^{-1}(p \mid v)$ is the inverse chi-square cumulative distribution function (CDF) for a given probability $p$ and $v$ degrees of freedom.

Proof: From (2), (3) and (5), we know that $F^{-1}(p \mid v)$ is directly proportional to $p$ for any given $v$. Let $\xi=$ $F^{-1}\left(1-\frac{N T}{D} \mid 2 n_{t r}\right)$. For $p_{\text {out }} \in\left(0,1-\frac{(N-1) T}{D}\right]$, we have

$$
\begin{aligned}
& \lim _{p_{\text {out }} \rightarrow 0} \bar{C}_{\text {out }}=0, \\
& \lim _{\text {pout }_{\text {ou }} \rightarrow \frac{N T}{D}} \bar{C}_{\text {out }}=\lambda_{3}\left(1-p_{\text {out }}\right) \log _{2}\left(1+\frac{\Lambda P_{c t} \xi}{n_{\text {tr }} \sigma^{2}}\right)
\end{aligned}
$$

Since $\lim _{p_{\text {out }} \rightarrow 1-\frac{N T}{D}} \bar{C}_{\text {out }}>0$ and (5) is continuous at $p_{\text {out }} \in$ $\left(0,1-\frac{(N-1) T}{D}\right]$, according to the extreme value theorem, there exists $p_{\text {out }} \in\left(0,1-\frac{(N-1) T}{D}\right]$ that can maximize $\bar{C}_{\text {out }}$.

\section{Energy Consumption Analysis}

For the transmission in each hop, the total power consumption is given by:

$$
P_{t}=\lambda_{1} P_{D P C O}+\lambda_{2} P_{b t}+\lambda_{3} P_{c t}+n_{t r} P_{c i r},
$$

where $P_{D P C O}$ is the power consumption of the DPCO scheme in the PP, $P_{\text {cir }}$ is the circuit power consumption of the sensor and $n_{t r}$ is the number of sensors used for transmission. The total energy consumption for the transmission through $N-1$ hops is give by:

$$
E_{t}=P_{t} \bar{T}=\frac{(N-1) P_{t} T}{1-p_{\text {out }}}
$$

In the $\mathrm{BP}$, we assume the channel is AWGN with free space path loss (i.e., $\alpha=2$ ). Thus given the transmission data rate $R_{b t}$, we have

$$
P_{b t}=r^{2} \sigma^{2}\left(2^{\frac{R_{b t}}{\lambda_{2}}}-1\right),
$$

where $r$ is the intracluster transmission range. We assume that the average cluster range is $r_{c}$ and $n$ sensors are normally deployed in each cluster. Therefore the approximate number of sensors that can successfully decode the broadcast message is given by:

$$
n_{t r}=\left(\frac{r}{r_{c}}\right)^{2} n=\frac{P_{b t} n}{r_{c}^{2} \sigma^{2}\left(2^{\frac{R_{b t}}{\lambda_{2}}}-1\right)} .
$$

In the $\mathrm{CP}$, a slow flat Rayleigh fading channel is assumed in the channel model. From (4) and (5), we have

$$
P_{c t}=\frac{\gamma_{\text {out }} n_{t r} \sigma^{2}}{\Lambda F^{-1}\left(p_{\text {out }} \mid 2 n_{\text {tr }}\right)}=\frac{2^{\frac{\bar{C}_{\text {out }}}{\text { (1- } \left._{\text {out }}\right)}}-1}{\Lambda F^{-1}\left(p_{\text {out }} \mid 2 n_{t r}\right)} n_{t r} \sigma^{2} \text {. }
$$


From (19), we can see that there is no closed-form solution for cooperative transmission power. In the next section, we propose a dynamic power control and optimization (DPCO) scheme to determine the optimal value of $n_{t r}, P_{b t}$ and $P_{c t}$ for the total energy consumption. We further propose an approximation algorithm for the DPCO scheme that can reduce its computational complexity.

\section{DynAmic POWER CONTROL AND Optimization SCHEME}

\section{A. Scheme Description}

For each single-hop transmission, the DPCO scheme is running in the PP. Given any QoS requirement with the delay $D>(N-1) T$, we aim to find the optimal value of $P_{c t}, P_{b t}, n_{t r}$ and $p_{\text {out }}$ for each single-hop transmission and thus minimize the total energy consumption of the multihop transmission (16). Note that $n_{t r}$ is directly related to $P_{b t}$ given the value of $R_{b t}$ in (18). Thus $n_{t r}$ and $P_{b t}$ are jointly optimized. From Proposition 2 and (19), we know that given $n_{t r}$, the transmission power $P_{c t \mid n_{t r}}$ is directly proportional to the maximum average outage capacity $\bar{C}_{\text {out }}^{*}$. According to the QoS requirement, we have $\bar{C}_{\text {out }} \geq \frac{L}{T}$ and $p_{\text {out }} \leq 1-\frac{(N-1) T}{D}$. $P_{c t \mid n_{t r}}$ is optimized when $p_{\text {out }}\left(n_{t r}\right)=p_{\text {out }}^{*}\left(n_{t r}\right)$ and $\bar{C}_{\text {out }}^{*}=$ $\frac{L}{T}$. Therefore, $P_{c t}$ and $p_{\text {out }}$ are also jointly optimized. The optimization problem can now be denoted as:

$$
\left\{P_{c t \mid n_{t r}^{*}}^{*}, n_{t r}^{*}\right\}=\underset{P_{c t \mid n_{t r}}, n_{t r}}{\arg \min } E_{t}\left(P_{c t \mid n_{t r}}, n_{t r}\right)
$$

Note that the value of $P_{c t \mid n_{t r}^{*}}^{*}$ depends on the choice of $n_{t r}^{*}$. However in order to determine $n_{t r}^{*}$, we need to figure out $P_{c t \mid n_{t r}}^{*}$ for every possible $n_{t r} . P_{c t \mid n_{t r}}$ and $n_{t r}$ are interrelated with each other in the optimization process.

In the DPCO scheme, we first find the conditional optimal $P_{c t \mid v}^{*}$ for all possible $v$, which is given by:

$$
\begin{aligned}
& P_{c t \mid v}^{*}=\underset{P_{c t \mid v}}{\arg \min } E_{t}\left(P_{c t \mid v} \mid v\right), 1 \leq v \leq n \\
& \text { s.t. } \quad P_{c t, \text { min }} \leq P_{c t \mid v} \leq P_{c t, \max },
\end{aligned}
$$

where $P_{c t, \text { min }}$ and $P_{c t, \max }$ are the minimum and maximum value of $P_{c t \mid v}$ respectively.

Then we can determine the optimal $n_{t r}^{*}$ as

$$
n_{t r}^{*}=\underset{v}{\arg \min } E_{t}\left(v \mid P_{c t \mid v}^{*}\right), 1 \leq v \leq n .
$$

We then have the optimal $P_{c t \mid n_{t r}^{*}}^{*}, P_{b t \mid n_{t r}^{*}}^{*}$ and thus the optimal $E_{t}^{*}$ according to (15) and (16).

Since there is no closed-form solution for cooperative transmission power $P_{c t \mid v}$, we use a dynamic power control (DPC) algorithm to find the conditional optimal $P_{c t \mid v}^{*}$. We will show that given any $n_{t r}=v, P_{c t \mid v}$ converges to its optimum $P_{c t \mid v}^{*}$ with a sufficient small change $\delta$ in $O(1)$ iterations.

In Algorithm 1, we use $P_{c t \mid v, I_{\max }}$ as the approximation of $P_{c t \mid v}^{*}$ since $\left|P_{c t \mid v, I_{\max }}-P_{c t \mid v}^{*}\right| \leq \delta$ where $\delta$ is sufficient small. For energy saving purpose, there is no need to cooperatively transmit the data if the QoS requirement cannot be fulfilled with the maximum transmission power $P_{c t, \max }$.

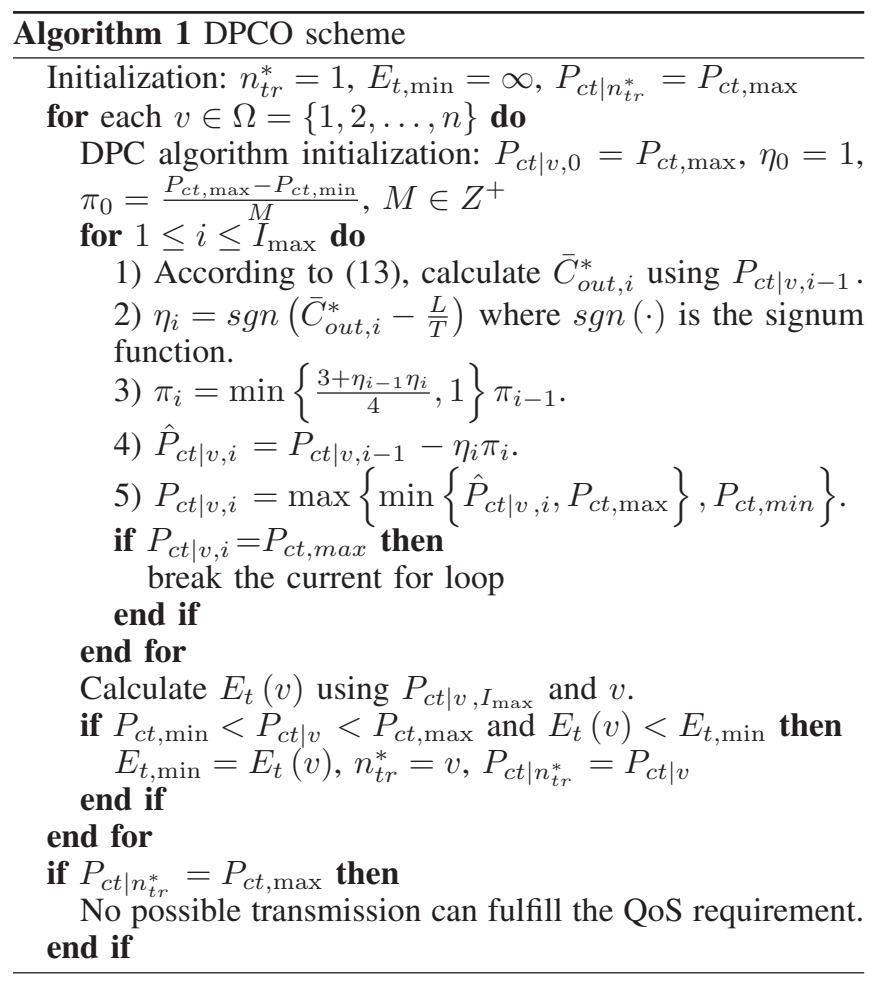

Proposition 3: Given $n_{t r}=v$ in the DPC algorithm, $P_{c t \mid v}$ converges to $P_{c t \mid v}^{*}$ with a sufficient small change $\delta$ in $O(1)$ iterations and we can use $P_{c t \mid v, I_{\max }}$ as the approximation of $P_{c t \mid v}^{*}$.

Proof: If $P_{c t, \min }<P_{c t \mid v}<P_{c t, \max }, P_{c t \mid v}^{*}$ must fall into one of the $M$ equal parts in interval $\left[P_{c t, \text { min }}, P_{c t, \text { max }}\right]$. We also notice that $\pi_{i}$ will not halve its value until $\eta_{i-1} \eta_{i}=-1$. Thus we have $M$ iterations at most before $\pi_{i}$ halves its value. After the first time $\pi_{i}$ is decreased by half, it is easy to see that $\pi_{i}$ can only stay unchanged for two iterations at most. We define $\delta$ as

$$
\delta=\frac{\pi_{0}}{2^{K}}=\frac{P_{c t, \max }-P_{c t, \min }}{2^{K} M}
$$

where $K$ is a positive integer that makes $\delta$ sufficient small compared with $\pi_{0}$. According to the algorithm it is obvious that $\left|P_{c t \mid v, i}-P_{c t \mid v}^{*}\right| \leq \pi_{i}$ after $\pi_{i}$ first halves itself. Thus we have $\left|P_{c t \mid v, i}-P_{c t \mid v}^{*}\right| \leq \delta$ in $O(M+2 K)=O(1)$ iterations. Let $I_{\max }>M+2 K$ and we can then use $P_{c t \mid v, I_{\max }}$ as the approximation of $P_{c t \mid v}^{*}$.

We assume that $n$ sensors are normally deployed in each cluster with the cluster range $r_{c}$. Since the minimum value of $R_{b t}$ is $\frac{L}{T}$, given $n_{t r}=v$, the optimal broadcast transmission power $P_{b t \mid v}^{*}$ is denoted as

$$
P_{b t \mid v}^{*}=\frac{v r_{c}^{2} \sigma^{2}\left(2^{\frac{L}{\lambda_{2} T}}-1\right)}{n} .
$$

After we have $P_{c t \mid v}^{*}$ and $P_{b t \mid v}^{*}$ for all possible $n_{t r}=v$, we can determine $n_{t r}^{*}$ according to (22) and thus in turn determine $P_{c t \mid n_{t r}^{*}}^{*}$ and $P_{b t \mid n_{t r}^{*}}^{*}$. 


\section{B. Approximation Algorithm}

To reduce the computational complexity in the DPCO scheme, we propose an approximation algorithm. Note that the computational complexity in DPCO scheme is mainly introduced by the DPC algorithm. The DPC algorithm is used for the optimization problem in (13) and (21) and there is no closed-form expression for $F^{-1}(p \mid v)$. If we can get an approximation of $F^{-1}(p \mid v)$ which can be quickly evaluated, the computational complexity will be significantly reduced. Inspired by the work in [9], we estimate the MISO channel in our model using a Gaussian approximation.

Since $\|H\|_{F}^{2}$ in (4) follows a chi-square variable with $2 n_{t r}$ degrees of freedom, we can calculate the mean value of $\gamma$ as:

$$
\mu_{\gamma}=\frac{\Lambda P_{c t}}{n_{t r} \sigma^{2}} E\left[\|H\|_{F}^{2}\right]=\frac{2 \Lambda P_{c t}}{\sigma^{2}} .
$$

We also calculate the variance of $\gamma$ as:

$$
\sigma_{\gamma}^{2}=\left(\frac{\Lambda P_{c t}}{n_{t r} \sigma^{2}}\right)^{2} \operatorname{var}\left(\|H\|_{F}^{2}\right)=\frac{1}{n_{t r}}\left(\frac{2 \Lambda P_{c t}}{\sigma^{2}}\right)^{2} .
$$

If we expand (2) in Taylor series at $\mu_{\gamma}$ we have

$$
C_{\text {out }}(\gamma)=\lambda_{3} \log _{2}\left(1+\mu_{\gamma}\right)-\sum_{k=1}^{\infty} \frac{\lambda_{3}}{k \ln 2}\left(\frac{\mu_{\gamma}-\gamma}{1+\mu_{\gamma}}\right)^{k} \text {. }
$$

According to (27) the second-order approximation for $\mu_{C}$ is given by

$$
\mu_{C}=E\left[C_{\text {out }}\right] \approx \lambda_{3} \log _{2}\left(1+\mu_{\gamma}\right)-\frac{\lambda_{3}}{2 \ln 2}\left(\frac{\sigma_{\gamma}}{1+\mu_{\gamma}}\right)^{2}
$$

where $\sigma_{\gamma}^{2}$ is the variance of $\gamma$. Note that the approximation is only valid for $\left|\gamma-\mu_{\gamma}\right|<1$. If we expand $C_{\text {out }}^{2}$ in a Taylor series, the second-order approximation for $\sigma_{C}^{2}$ is given by:

$$
\begin{aligned}
\sigma_{C}^{2} & =E\left[C_{\text {out }}^{2}\right]-\left(E\left[C_{\text {out }}\right]\right)^{2} \\
& \approx\left(\frac{\lambda_{3}}{\ln 2}\right)^{2}\left[\frac{\sigma_{\gamma}^{2}}{\left(1+\mu_{\gamma}\right)^{2}}-\frac{\sigma_{\gamma}^{4}}{4\left(1+\mu_{\gamma}\right)^{4}}\right] .
\end{aligned}
$$

We assume that the approximate channel capacity $\tilde{C}_{\text {out }}$ follows a normal distribution with a mean $\mu_{C}$ and a standard deviation $\sigma_{C}$, which is denoted as $\tilde{C}_{\text {out }} \sim N\left(\mu_{C}, \sigma_{C}^{2}\right)$. We further define the outage probability $\tilde{p}_{\text {out }}$ for $\tilde{C}_{\text {out }}$, which is given by:

$$
\tilde{p}_{\text {out }}=\operatorname{Pr}\left(C<\tilde{C}_{\text {out }}\right)=\frac{1}{2}+\frac{1}{2} \operatorname{erf}\left(\frac{\tilde{C}_{\text {out }}-\mu_{C}}{\sqrt{2} \sigma_{C}}\right)
$$

where $\operatorname{erf}(\cdot)$ is the error function. From (30) we have

$$
\tilde{C}_{\text {out }}=\mu_{C}+\sqrt{2} \sigma_{C} \operatorname{erf}^{-1}\left(2 \tilde{p}_{\text {out }}-1\right)
$$

where $\operatorname{erf}^{-1}(\cdot)$ is the inverse error function, which can also be defined in terms of the Maclaurin series.

$$
\operatorname{erf}^{-1}(z)=\frac{\sqrt{\pi}}{2}\left(z+\frac{\pi}{12} z^{3}+\frac{7 \pi^{2}}{480} z^{5}+\cdots\right) .
$$

The approximate average outage capacity is defined as

$$
\begin{aligned}
\bar{\sim}_{\text {out }} & =\left(1-\tilde{p}_{\text {out }}\right) \tilde{C}_{\text {out }} \\
& \approx \frac{1-\tilde{p}}{2}\left(\mu_{C}+\sqrt{\frac{\pi}{2}} \sigma_{C}\left(\tilde{p}+\frac{\pi}{12} \tilde{p}^{3}+\frac{7 \pi^{2}}{480} \tilde{p}^{5}\right)\right)
\end{aligned}
$$

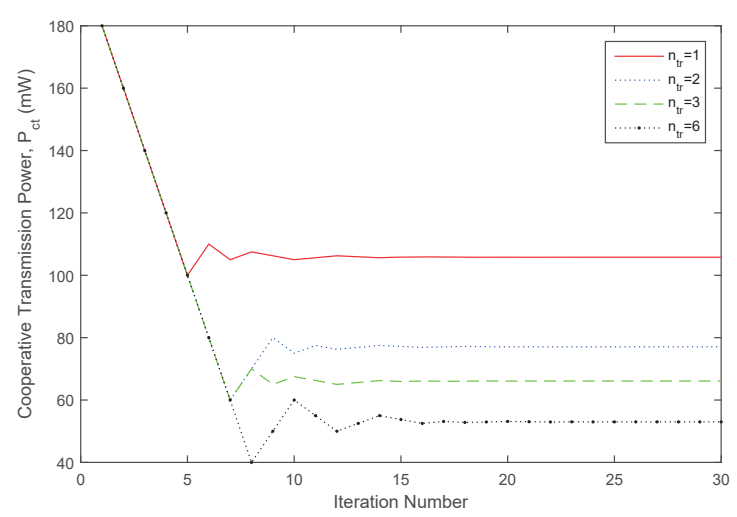

Fig. 2. Performance of the dynamic power control algorithm with different $n_{t r}$

where $\tilde{p}=2 \tilde{p}_{\text {out }}-1$. Compared with (13), we can see that (33) is a polynomial function on $\tilde{p}$ that has a straightforward solution for the optimization of $\overline{\tilde{}} C_{\text {out }}$. If we substitute $\overline{\tilde{}} C_{\text {out }}$ for $\bar{C}_{\text {out }}$ and $\tilde{p}_{\text {out }}$ for $p_{\text {out }}$ respectively, the computational complexity in the first step of the DPC algorithm can be significantly reduced.

\section{Simulation AND ANALysis}

In this section, we give the simulation results to show the efficiency of DPCO scheme. We also show that the approximation algorithm provides accurate channel estimations. The parameters in the simulation are given in TABLE I.

TABLE I. PARAMETERS FOR SIMULATION

\begin{tabular}{|l|l|l|}
\hline Symbol & Description & Value \\
\hline$\beta$ & Path loss constant & 1 \\
\hline$\alpha$ & Path loss exponent & 2 \\
\hline$n$ & Number of sensors per cluster & 10 \\
\hline$d$ & Transmission distance & $100 \mathrm{~m}$ \\
\hline$r_{c}$ & Cluster range & $10 \mathrm{~m}$ \\
\hline$\sigma^{2}$ & Noise power & $5 \mu \mathrm{W}$ \\
\hline$P_{c t, \max }$ & Maximum of $P_{c t}$ & $200 \mathrm{~mW}$ \\
\hline$P_{c t, \min }$ & Minimum of $P_{c t}$ & $0 \mathrm{~mW}$ \\
\hline$\pi_{0}$ & Initial step in DPC algorithm & $10 \mathrm{~mW}$ \\
\hline$P_{c i r}$ & Circuit power consumption & $10 \mathrm{~mW}$ \\
\hline$\frac{L}{T}$ & Capacity Requirement & $1 \mathrm{bps} / \mathrm{Hz}$ \\
\hline$\lambda_{1}, \lambda_{2}, \lambda_{3}$ & & $0.1,0.15,0.75$ \\
\hline
\end{tabular}

First, we investigate the DPC algorithm. We set $M=20$ and $\pi_{0}=10 \mathrm{~mW}$. Given different $n_{t r}=v$, the simulation results in Fig. 2 show that $P_{c t \mid v}$ converges to its optimal value $P_{c t \mid v}^{*}$ with sufficiently small difference in a limited number of iterations $\left(I_{\max }=30\right)$. Note that the optimal cooperative transmission power $P_{c t \mid n_{t r}}^{*}$ is inversely proportional to the number of cooperative sensors $n_{t r}$.

In Fig. 3 we show that under the QoS requirement, there exists the optimal outage probability $p_{\text {out }}^{*}$ that can maximize the average outage capacity $\bar{C}_{\text {out }}$. Given $n_{t r}=6$, Fig. 3 shows that $\bar{C}_{\text {out }}$ reaches its maximum value $\bar{C}_{\text {out }}^{*}=1 \mathrm{bps} / \mathrm{Hz}$ at $p_{\text {out }}^{*}=0.188$. We also see that the approximation algorithm gives a similar result to that for the actual channel. In this case, the approximate average outage capacity $\overline{ }^{C} C_{\text {out }}$ reaches its maximum value $\overline{\tilde{C}} C_{\text {out }}^{*}=0.998 \mathrm{bps} / \mathrm{Hz}$ at $\tilde{p}_{\text {out }}^{*}=0.178$. 


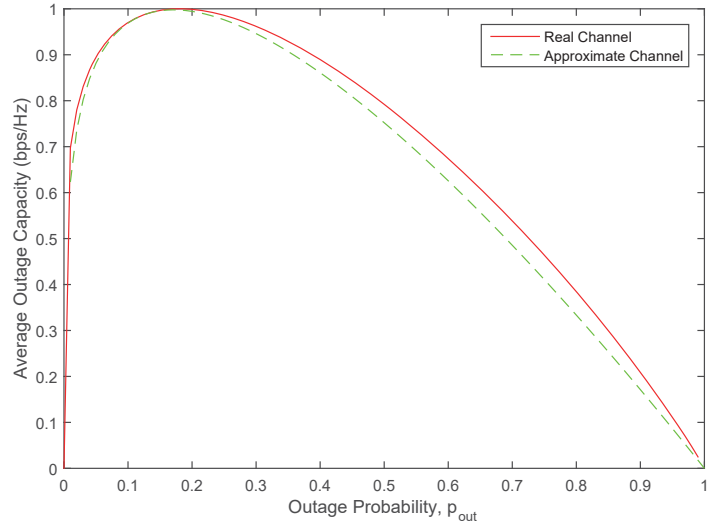

Fig. 3. Average outage capacity with different $p_{\text {out }}$ for $n_{t r}=6$ and $P_{c t \mid n_{t r}}=53 \mathrm{~mW}$

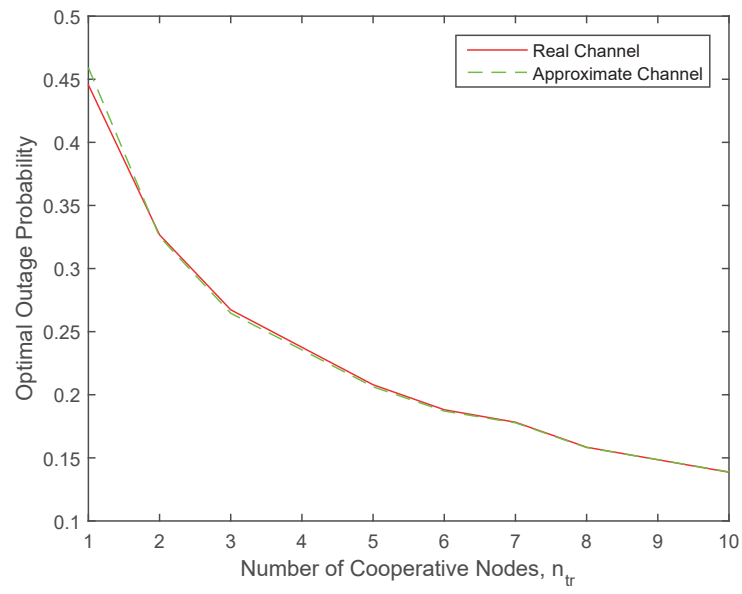

Fig. 4. Optimal outage probability with different $n_{t r}$

We give the optimal outage probability $p_{\text {out }}^{*}$ for various values of $n_{t r}$ in Fig. 4. It shows that $p_{\text {out }}^{*}$ is inversely proportional to $n_{t r}$. We also note that the approximation algorithm gives a similar result.

The relation of $P_{t}$ and $n_{t r}$ is given in Fig. 5. From (15) and taking into account the retransmissions, we define the average total power consumption as

$$
\bar{P}_{t}\left(n_{t r}\right)=\frac{P_{t}\left(n_{t r}\right)}{1-p_{\text {out }}^{*}\left(n_{t r}\right)}
$$

where $p_{\text {out }}^{*}\left(n_{t r}\right)$ is the optimal outage probability corresponding to $n_{t r}$. From Fig. 5, we see that $P_{t}$ and $\bar{P}_{t}$ reach their minimum value at $n_{t r}=2$ and $n_{t r}=3$ respectively. We choose $n_{t r}=3$ as the optimal solution according to the definition in (16) and (34). Note that for the optimal $n_{t r}^{*}$, the optimal outage probability $p_{\text {out }}^{*}\left(n_{t r}^{*}\right)$ should fulfill the QoS requirement $p_{\text {out }}^{*}\left(n_{t r}^{*}\right) \leq 1-\frac{(N-1) T}{D}$. If $p_{\text {out }}^{*}\left(n_{t r}^{*}\right)$ is out of range $\left(p_{\text {out }}^{*}\left(n_{t r}^{*}\right)>1-\frac{(N-1) T}{D}\right)$, the new optimal value $\hat{n}_{t r}^{*}$ should be the smallest $n_{t r} \in\left(n_{t r}^{*}, n\right]$ that fulfills the QoS requirement, since $\bar{P}_{t}$ monotonically increases with $n_{t r} \in\left(n_{t r}^{*}, n\right]$ and $p_{o u t}^{*}\left(n_{t r}^{*}\right)$ is inversely proportional to $n_{t r}$.

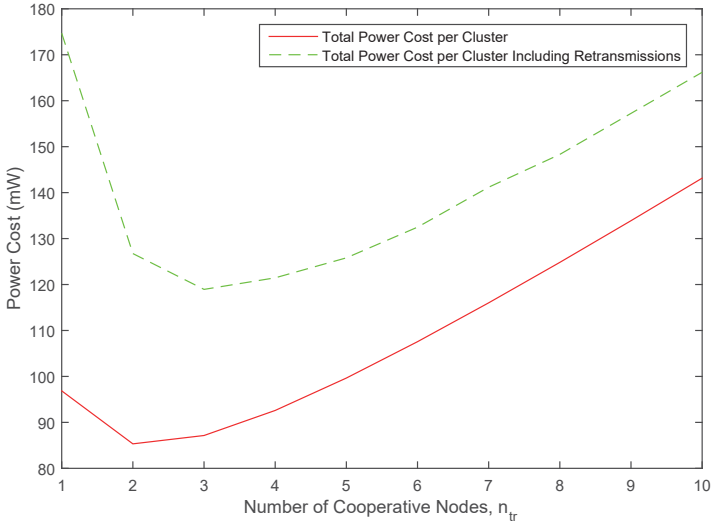

Fig. 5. Performance of the dynamic power control algorithm with different values of $n_{t r}$

\section{CONCLUSION}

In this paper, we investigated the power consumption and optimization in a multi-hop QoS-constrained cooperative WSN in a fading environment. We showed that there is no closed form solution for the power optimization problem with certain QoS constraints in the fading environment. For each singlehop transmission, we proposed a three-phase transmission scheme. We then proposed the DPCO scheme to optimize the power consumption. By using DPCO scheme, cooperative diversity is utilized and the power consumption of the multi-hop transmission is minimized. We further proposed a channel approximation algorithm to reduce the computational complexity of DPCO scheme. The simulation results showed the efficiency of the DPCO scheme.

\section{REFERENCES}

[1] V. Gungor and G. Hancke, "Industrial wireless sensor networks: Challenges, design principles, and technical approaches," Industrial Electronics, IEEE Transactions on, vol. 56, no. 10, pp. 4258-4265, Oct 2009.

[2] A. Paulraj, R. Nabar, and D. Gore, Introduction to Space-Time Wireless Communications, 1st ed. New York, NY, USA: Cambridge University Press, 2008.

[3] S. Cui, A. Goldsmith, and A. Bahai, "Energy-efficiency of mimo and cooperative mimo techniques in sensor networks," Selected Areas in Communications, IEEE Journal on, vol. 22, no. 6, pp. 1089-1098, Aug 2004.

[4] S. Jayaweera, "Virtual mimo-based cooperative communication for energy-constrained wireless sensor networks," Wireless Communications, IEEE Transactions on, vol. 5, no. 5, pp. 984-989, May 2006.

[5] A. Del Coso, U. Spagnolini, and C. Ibars, "Cooperative distributed mimo channels in wireless sensor networks," Selected Areas in Communications, IEEE Journal on, vol. 25, no. 2, pp. 402-414, February 2007.

[6] A. Goldsmith, Wireless Communications. New York, NY, USA: Cambridge University Press, 2005.

[7] O. Younis and S. Fahmy, "Heed: a hybrid, energy-efficient, distributed clustering approach for ad hoc sensor networks," Mobile Computing, IEEE Transactions on, vol. 3, no. 4, pp. 366-379, Oct 2004.

[8] W. Heinzelman, A. Chandrakasan, and H. Balakrishnan, "Energyefficient communication protocol for wireless microsensor networks," in System Sciences, 2000. Proceedings of the 33rd Annual Hawaii International Conference on, Jan 2000, pp. 10 pp. vol.2-.

[9] J. Perez, J. Ibanez, L. Vielva, and I. Santamaria, "Closed-form approximation for the outage capacity of orthogonal stbc," Communications Letters, IEEE, vol. 9, no. 11, pp. 961-963, Nov 2005. 